\title{
Retrieving the Hemodynamic Response Function in resting state fMRI : methodology and application
}

\author{
Guo-Rong Wu, Gopikhrishna Deshpande, Steven Laureys and Daniele Marinazzo
}

\begin{abstract}
In this paper we present a procedure to retrieve the hemodynamic response function (HRF) from resting state functional magnetic resonance imaging (fMRI) data. The fundamentals of the procedures are further validated by considering simultaneous electroencephalographic (EEG) recordings. The typical HRF shape at rest for a group of healthy subject is presented. Then we present the modifications to the shape of the HRF at rest following two physiological modulations: eyes open versus eyes closed and propofol-induced modulations of consciousness.
\end{abstract}

\section{INTRODUCTION}

Functional magnetic resonance imaging (fMRI) time series can be modeled as a convolution of a latent neural signal (which is not measured) and the hemodynamic response function (HRF). First, since the temporal characteristics of the HRF across different anatomical regions can be influenced by the underlying venous structure, it is possible that intrinsic activity across disparate brain regions can be temporally correlated only due to the underlying vascular architecture. Second, the hemodynamic response is affected by physiological fluctuations arising from cardiac pulsation and respiration [1]. These can introduce temporal correlations into resting state (RS) fMRI signals. Also, given the fact that RS-fMRI data is sampled slowly (typically every 1-2 seconds), physiological fluctuations cannot be removed by simple filtering as they can alias into the low frequency band of interest $(0.01-0.1 \mathrm{~Hz})$. Third, the period of the fastest variation in RS-fMRI data is $10 \mathrm{~s}$, which is orders of magnitude greater than the sub-second time scale at which most neuronal processes occur.

This confounding effect can be dealt with by deconvolution of the HRF. In task-related fMRI this procedure has been known and applied since the very beginnings, since the onset of the HRF was known. This is not the case for RS-fMRI.

Motivated by this evidence, we developed an approach to perform blind hemodynamic deconvolution [2] of RS-fMRI data to recover the underlying latent neuronal signals. This allowed to greatly improve the estimation of directed

G.-R. Wu is with the Department of Data Analysis, University of Ghent, B9000 Ghent, Belgium and the Key Laboratory of Cognition and Personality, Southwest University, Chongqing, China (e-mail: gronwu@gmail.com).

G. Deshpande is with the Department of Electrical and Computer Engineering, Auburn University, Auburn, AL 36849, USA (e-mail: gopi@auburn.edu).

S. Laureys is with the Coma Science Group, Cyclotron Research Centre, GIGA - Research Center and Department of Neurology, University of Liège, Liège, (email: steven.laureys@ulg.ac.be)

D. Marinazzo is with the Department of Data Analysis, University of Ghent, B9000 Ghent, Belgium (tel: +3292646375, e-mail: daniele.marinazzo@ugent.be). dynamical influences in RS-fMRI recordings [2], but also provided us with the estimation of the HRF shape for each voxel in the brain. In this paper we will first explore the coupling between electrically measured brain activity and HRF using simultaneous electroencephalographic (EEG) recordings, then we analyzed the effects of physiological conditions (eyes open vs. eyes closed and modulations of consciousness) on the HRF shape.

\section{Methodology}

The deconvolution is blind because there is no external input in case of RS-fMRI data and consequently, both the HRF and the underlying neuronal latent variables must be simultaneously estimated from the observed fMRI data, making this an ill-posed estimation problem.

The blind hemodynamic deconvolution of resting state data was performed using the approach proposed by $\mathrm{Wu}$ et al [2]. Specifically, neural events were detected in the resting BOLD signal as point processes corresponding to signal fluctuations with a given signature [3], specifically individuated when the standardized BOLD signal crossed a given threshold. These pseudo-events were then aligned in order to evaluate the exact delay between a pseudo-event at cortical level and the BOLD signature. This procedure allowed to extract voxel specific HRF and perform Wiener deconvolution of the BOLD signal [4]. The HRF can be reconstructed in several ways: the canonical shape (with time and space derivatives), as a Finite Impulse Response (FIR) or 'selective averaging' [5].

The procedure described above is sketched in Figure 1.

Figure 2 reports the typical HRF parameters (Height, Time to Peak and Full Width at Half Maximum) for a pool of 32 healthy subjects, as described in [2]. It is worth to note how the variations in HRF are consistent with the differences in net arterial and venous flow, and the consequent effects on the estimation of Granger causality reported in [6]. This confirms the importance of performing HRF deconvolution prior to estimating lag-based directed connectivity.

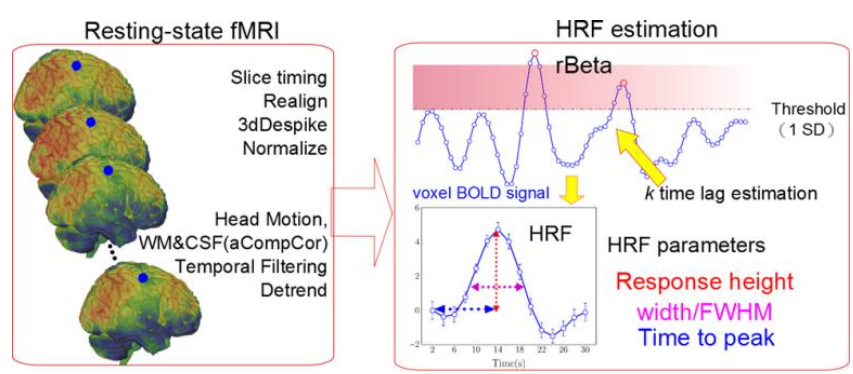

Figure $1:$ scheme of the procedure 


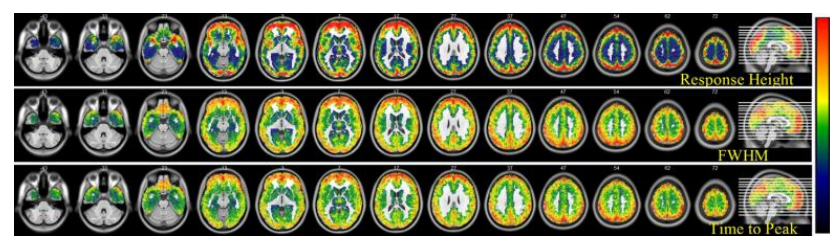

Figure 2 : parameters of the HRF retrieved at rest

\section{APPLICATIONS}

\section{A. Relation with EEG power}

In order to further investigate the electrophysiological basis of the HRF and its coupling to electrical brain activity we considered simultaneously recorded EEG and fMRI data. EEG were collected at $1000 \mathrm{~Hz}$ and down-sampled at $250 \mathrm{~Hz}$. Scanner artifact correction, pulse artifact correction, notch filtering and ICA analysis were performed on the raw data. fMRI data were collected at 7 Tesla, with a repetition time of 1s. Resting-state fMRI data preprocessing was carried out using both AFNI and SPM8 package. First, the EPI volumes were corrected for the temporal difference in acquisition among different slices, and then the images were realigned to the first volume for head-motion correction. The resulting volumes were then despiked using AFNI's 3dDespike algorithm to mitigate the impact of outliers. Next, the despiked images were spatially normalized to the Montreal Neurological Institute template then resampled to 3-mm isotropic voxels.

Several parameters were included in a linear regression to remove possible spurious variances from the data. These were i) six head motion parameters obtained in the realigning step, ii)non-neuronal sources of noise estimated using the anatomical component correction method (aCompCor, the representative signals of no interest from white matter (WM) and cerebral spinal fluid (CSF) included the top five principal components (PCs) from WM and the top five from CSF mask; the subject-specific WM and CSF masks was segmented from the anatomical image of each participant using SPM8's unified segmentation-normalization procedure) [7]. Then the time series were temporally band-pass filtered $(0.01-0.08 \mathrm{~Hz})$ and linearly detrended.

The scalp EEG voltage data from the three occipital channels $\mathrm{O} 1, \mathrm{O} 2$, and $\mathrm{Oz}$ were selected.

First, EEG signals for each channel were segmented into $500 \mathrm{~ms}$ non-overlapping epochs. Second, the EEG power spectrum for each single epoch was calculated using a nonparametric multitaper approach, and the alpha band power was obtained by integrating the power spectrum between 8 and $12 \mathrm{~Hz}$. Third, the channel-level alpha power time series from each of the three occipital channels was averaged to yield the subject-level alpha power time series, which was convolved with a canonical hemodynamic response function (HRF). The HRF-convolved alpha power time series was then downsampled to the same sampling frequency as the BOLD signal.

To identify brain regions whose BOLD activity co-varied with EEG alpha power, we examined the temporal correlation between HRF-convolved alpha power time series and BOLD time series from all voxels based on the general linear model (GLM). HRF-convolved alpha power time series was incorporated as a parametric regressor in the GLM, modeling the coupling effects between alpha and BOLD .

The processed BOLD signal at every voxel was converted into its $\mathrm{z}$-score, and the resting state HRF was retrieved as described above, according to the selective averaging procedure.

Two canonical ROIs were chosen from the previous GLM analysis (Thalamus and Occipital Lobe) both for eye closed and open condition, under individual voxel $p$-value $<1 \mathrm{E}-6$, cluster size $>50$. A positive correlation between deconvolved BOLD and EEG filtered in alpha band was observed in the thalamus, and a negative one in the Occipital Lobe (Figure 3).
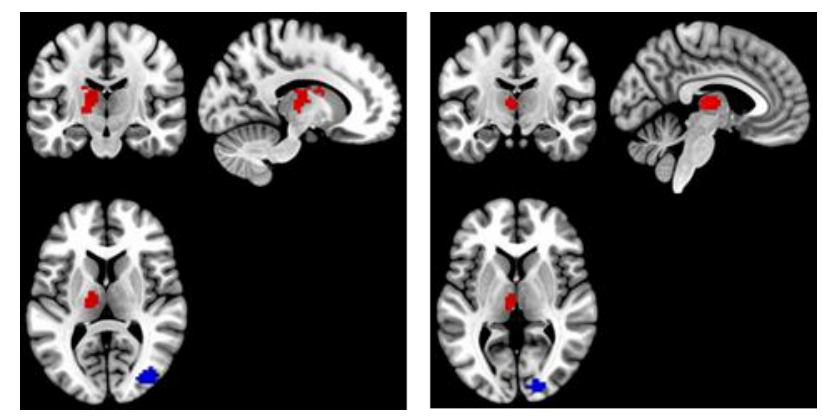

Figure 3 : clusters of significant correlation (red) and anticorrelation (blue) between deconvolved BOLD and alpha power spectrum

The typical HRF shapes derived in these two regions in the two conditions are reported in Figure 4. Interestingly we observe two subfamilies of HRF shapes in the thalamus. Their nature and function will need to be investigated further.
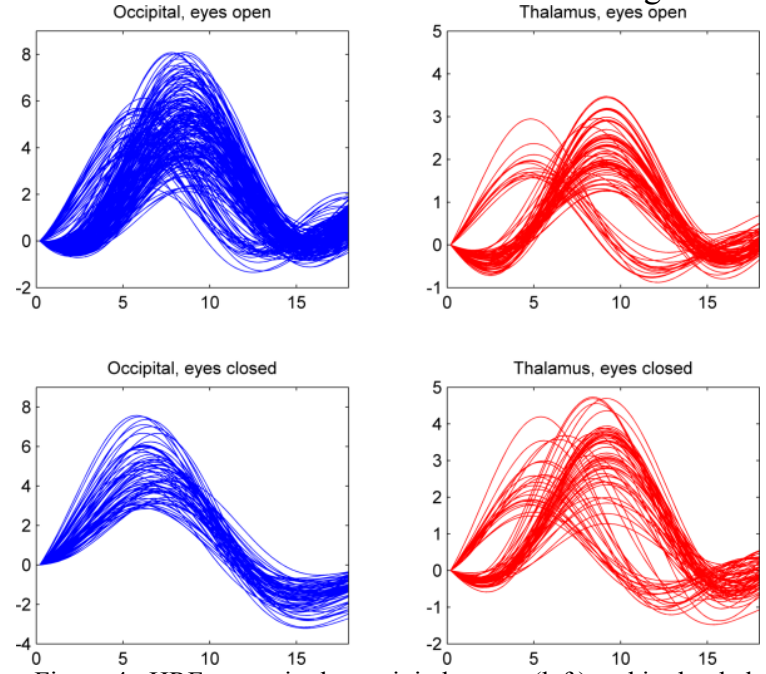

Figure $4: \mathrm{HRF}$ at rest in the occipital cortex (left) and in the thalamus (right) for eyes open and closed

\section{B. HRF modulations with eyes open and closed}

In order to study the modulations of HRF shape when opening or closing the eyes on a larger sample, we considered a data set of 48 healthy controls collected at 
the Beijing Normal University in China with 3 resting state fMRI scans of six minutes each. During the first scan participants were instructed to rest with their eyes closed. The second and third resting state scan were randomized between resting with eyes open versus eyes closed. In addition this dataset contains a 64-direction DTI scan for every participant. Data were preprocessed as described above. Using a contrast taking into account the three conditions (eyes closed, open, closed again), we observed significant differences in the height of the HRF in the areas depicted in figure 5. The corresponding HRF shape is also reported.
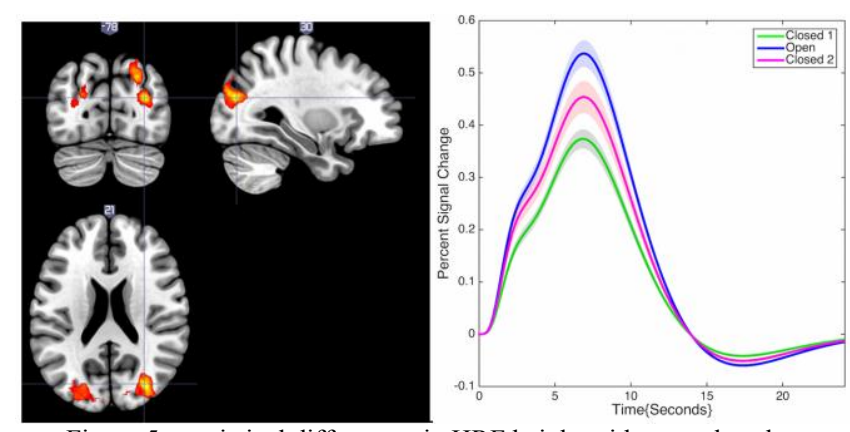

Figure 5 : statistical differences in HRF height with eyes closed, open then closed again (left), and typical shapes within the cluster (right)

\section{HRF modulations in induced loss of consciousness}

Twenty one healthy right-handed volunteers (age range, $18-34$ yr; mean age $\pm \mathrm{SD}, 23.4 \pm 4$.1yrs; 5 males) participated in the study. The subjects provided written informed consent to participate in the study. None of the healthy subjects had a history of head trauma or surgery, mental illness, drug addiction, asthma, motion sickness, or previous problems during anesthesia. The study was approved by the Ethics Committee of the Medical School of the University of Liege (University Hospital, Liege, Belgium).

Functional MRI acquisition consisted of resting-state fMRI volumes repeated in four clinical states only for healthy volunteers: normal wakefulness (W1), mild sedation (S1), deep sedation (S2), and recovery of consciousness (W2). The temporal order of mild- and deep-sedation conditions was randomized. The typical scan duration was half an hour in each condition. The number of scans per session was matched in each subject to obtain a similar number of scans in all four clinical states (mean $\pm \mathrm{SD}, 251 \pm 77$ scans/session).

Functional images were acquired on a 3 Tesla Siemens Allegra scanner (Siemens AG, Munich, Germany; Echo Planar Imaging sequence using 32 slices; repetition time $=2460 \mathrm{~ms}$, echo time $=40 \mathrm{~ms}$, field of view $=220 \mathrm{~mm}$, voxel size $=3.45 \times 3.45 \times 3 \mathrm{~mm}$, and matrix size $=64 \times 64 \times 32$ ). During data acquisition, subjects wore earplugs and headphones. The most comfortable supine position attainable was sought to avoid painful stimulation related to position. The same preprocessing procedure described above for the simultaneous EEG/fMRI data was applied also to these data.

A two-sample t-test with three covariates (age, gender and mean framewise displacement [8]) was implemented in SPM8 to map group difference between four levels of consciousness, independently for HRF parameters.

HRF parameters and the amount of spontaneous events for each subject individually were entered into a random-effects analysis (one-way ANOVA within subjects, with three covariate (age, gender and mean displacement) to identify regions which showed significant activation differences among four clinical states), a linear $\mathrm{T}$ contrast was computed, searching for a linear relationship between HRF and the level of consciousness of the subjects across the four conditions ( contrast (W1 W2 S1 S2) [1.5 $0.5-1.5-0.5]$ ).

Type I error due to multiple comparisons across voxels was controlled by false discovery rate method [9]. Statistical significance for group analysis was set at $\mathrm{P}_{\mathrm{FDR}}<0.05$, derived from the Gaussian random field theory.

As reported in figure 6, we observed statistical differences in all the three HRF parameters, as well as in the number of spontaneous events, mainly in frontal areas which are reported to be implicated in modulations of consciousness from traditional fMRI studies. The corresponding HRF shape is also reported.
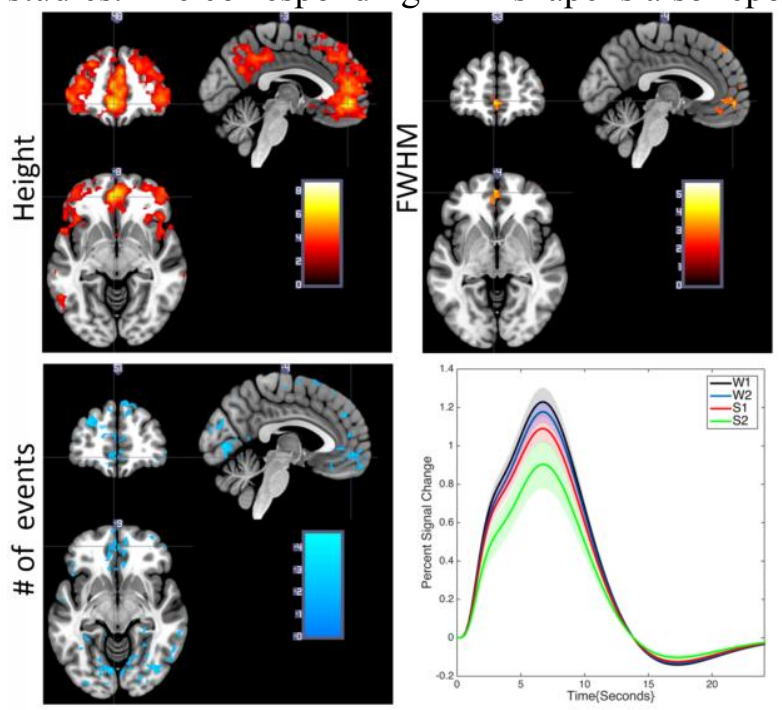

Figure 6 : statistics on pseudoevents and resting HRF parameters th a contrast following the level of consciousness, and typical shapes within the cluster (bottom right) 


\section{CONCLUSIONS AND FUTURE WORK}

We have presented a methodology to retrieve the hemodynamic response function at from resting state functional magnetic resonance imaging data. The results are promising since the shape of the retrieved HRF is consistent with the literature and supports evidences of the vascular flow. Additionally, the functional modifications to the HRF shape are consistent with evidence previously reported using different methodologies. The approach will need further validation using electrophysiological and cardiovascular data.

\section{REFERENCES}

[1] D. Cordes, V. M. Haughton, K. Arfanakis, J. D. Carew, P. A. Turski, C. H. Moritz, et al., "Frequencies contributing to functional connectivity in the cerebral cortex in "resting-state" data," AJNR Am J Neuroradiol, vol. 22, pp. 1326-33, Aug 2001.

[2] G. R. Wu, W. Liao, S. Stramaglia, J. R. Ding, H. Chen, and D. Marinazzo, "A blind deconvolution approach to recover effective connectivity brain networks from resting state fMRI data," Med Image Anal, vol. 17, pp. 365-74, Apr 2013.

[3] E. Tagliazucchi, P. Balenzuela, D. Fraiman, and D. R. Chialvo, "Criticality in large-scale brain FMRI dynamics unveiled by a novel point process analysis," Front Physiol, vol. 3, p. 15, 2012.

[4] G. H. Glover, "Deconvolution of impulse response in event-related BOLD fMRI," Neuroimage, vol. 9, pp. 416-29, Apr 1999.

[5] A. M. Dale and R. L. Buckner, "Selective averaging of rapidly presented individual trials using fMRI," Hum Brain Mapp, vol. 5, pp. 329-40, 1997.

[6] J. T. Webb, M. A. Ferguson, J. A. Nielsen, and J. S. Anderson, "BOLD Granger causality reflects vascular anatomy," PLoS One, vol. 8, p. e84279, 2013.

[7] Y. Behzadi, K. Restom, J. Liau, and T. T. Liu, "A component based noise correction method (CompCor) for BOLD and perfusion based fMRI," Neuroimage, vol. 37, pp. 90-101, Aug 12007.

[8] J. D. Power, K. A. Barnes, A. Z. Snyder, B. L. Schlaggar, and S. E. Petersen, "Spurious but systematic correlations in functional connectivity MRI networks arise from subject motion," Neuroimage, vol. 59, pp. 2142-2154, 2012.

[9] J. Chumbley, K. Worsley, G. Flandin, and K. Friston, "Topological FDR for neuroimaging," Neuroimage, vol. 49, pp. 3057-64, Feb 152010. 